\title{
A Guess Model of Black Holes and the Evolution of Universe
}

\author{
Zhenhua Mei ${ }^{1}$, Shuyu $\mathrm{Mei}^{2}$ \\ ${ }^{1}$ College of Chemistry and Molecular Engineering, Qingdao University of Science and Technology, Qingdao, China \\ ${ }^{2}$ Medical College, Qingdao University, Qingdao, China \\ Email: mzh62@qust.edu.cn
}

Received June 10, 2012; revised July 16, 2012; accepted July 23, 2012

\begin{abstract}
Based on the gravitational theory, fundamental data, and comprehensible suppositions, an evolution model of the universe was proposed. The universe exists in explosion and constringency mobile equilibrium state. The critical sizes of celestial bodies were calculated in their evolution process.
\end{abstract}

Keywords: Black Hole; Evolution; Universe; Gravitation; Entropy

\section{Introduction}

As the theoretical ratiocination (Einstein's theory of relativity) and the cumulating of the more and more obtained data or phenomena from astronomic observation of the celestial bodies, people began to have a general impression of the universe, such as the clangorous words expressed: big bang, expanding universe, and black hole et al. (Ginsburg 1985). From a point of view that the microcosm decides the macrocosm, more information of the universe can be predicted from present knowledge of the elementary particles.

In this paper, some parameters of evolution of the universe were calculated. The main thoughts were the Newton's universal gravitation and the structures of matter.

\section{Preparation of Research}

\subsection{Fundamental Data and Suppositions}

The gravitational constant, $G$, is $6.670 \times 10^{-11} \mathrm{~N} \mathrm{~m}^{2} \cdot \mathrm{kg}^{-2}$. The radius of hydrogen atom, $a_{0}$, is $5.29 \times 10^{-11} \mathrm{~m}$. The mass density of atomic nucleus, $\rho_{n}$, was determined (Erdei 1976) to be $2 \times 10^{17} \mathrm{~kg} \cdot \mathrm{m}^{-3}$. The mass of neutron, $m_{n}$, is $1.67495 \times 10^{-27} \mathrm{~kg}$, radius $r_{n}, 1.2598 \times 10^{-15} \mathrm{~m}$. The combining energy of nucleus is $8 \mathrm{MeV}$. The gravitational acceleration on earth surface is $9.81 \mathrm{~m} \cdot \mathrm{s}^{-2}$. The sun has the mass of $1.989 \times 10^{30} \mathrm{~kg}$, density of $1.409 \times 10^{3}$ $\mathrm{kg} \cdot \mathrm{m}^{-3}$, radius of $6.960 \times 10^{8} \mathrm{~m}$.

Suppositions: the combining energy of nucleus (strong interaction in nucleus) came from gravitation, and the nucleus consisted of gravitons; the combinative form between nucleus was its overlaps one another.

\subsection{Formula Derivation A-Gravitational Acceleration of a Particle from Solid Sphere}

According to Newton's law, the gravitational force between two distant particles has the form:

$F=G m M / r^{2}$, and $F=m a$. Hence, the gravitational acceleration, $a=G M / r^{2}$.

However, to a neighboring large body (has a mass of $M$, radius of $R$ ), the radius of the body cannot be neglected; its accurate result can be obtained by processing a mathematical integral.

As Figure 1 expressed, a solid sphere has a radius of $R$, a homogeneous density of $\rho$, the particle has a mass, $\mathrm{m}$, and has a distance, $n R$, from the center, the gravitational acceleration, $a$, of the particle was derived as follows:

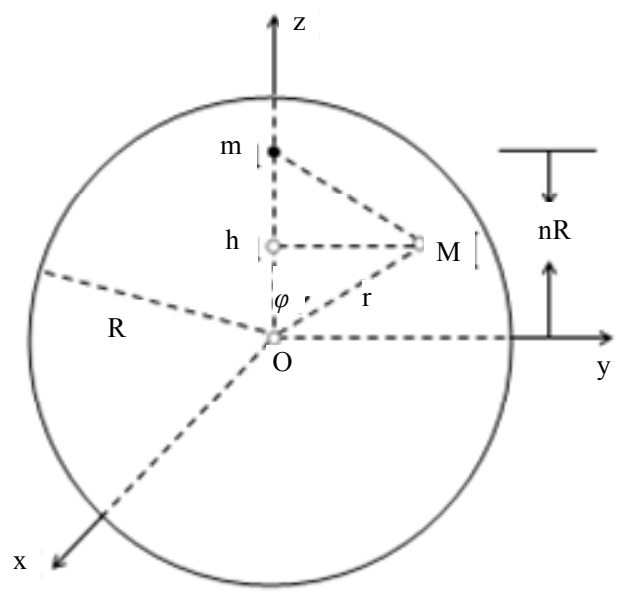

Figure 1. Sketch map of a particle (m), which with a distance, $n R$, from the center of the solid sphere. 


$$
\begin{aligned}
a & =\int_{\Omega} \mathrm{d} a=\int_{\Omega} \frac{G}{r^{2}} \mathrm{~d} M=\iiint_{\Omega} G \rho^{\prime} \frac{\overline{m h}}{\overline{m M}} \mathrm{~d} x \mathrm{~d} y \mathrm{~d} z=G \rho^{\prime} \iiint_{\Omega} \frac{n R-z}{\left(x^{2}+y^{2}+(n R-z)^{2}\right)^{3 / 2}} \mathrm{~d} x \mathrm{~d} y \mathrm{~d} z \\
& =4 G k \rho \int_{0}^{R} \mathrm{~d} x \int_{0}^{R} \mathrm{~d} y \int_{(n+1) R}^{(n-1) R} \mathrm{~d} t \mathrm{~d} \frac{-t}{\left(x^{2}+y^{2}+t^{2}\right)^{3 / 2}} \frac{t=n R-z}{\longrightarrow} \\
& =\left.4 G k \rho \int_{0}^{R} \mathrm{~d} x \int_{0}^{R} \mathrm{~d} y\left(x^{2}+y^{2}+t^{2}\right)^{-1 / 2}\right|_{(n+1) R} ^{(n-1) R} \\
& =4 G k \rho \int_{0}^{R} \mathrm{~d} x \int_{0}^{R} \mathrm{~d} y\left(\left(x^{2}+y^{2}+(n-1)^{2} R^{2}\right)^{-1 / 2}-\left(x^{2}+y^{2}+(n+1)^{2} R^{2}\right)^{-1 / 2}\right) \\
& =4 G k \rho \int_{0}^{R} \mathrm{~d} x\left(\ln \left(y+\sqrt{x^{2}+y^{2}+(n-1)^{2} R^{2}}\right) \ln \left(y+\sqrt{x^{2}+y^{2}+(n+1)^{2} R^{2}}\right)\right) \\
a & =4 G k \rho \int_{0}^{R} \mathrm{~d} x\left(\ln \left(R+\sqrt{x^{2}+R^{2}+(n-1)^{2} R^{2}}\right)-\ln \left(R+\sqrt{x^{2}+R^{2}+(n+1)^{2} R^{2}}\right)\right. \\
& +\left(\ln \left(\sqrt{x^{2}+(n+1)^{2} R^{2}}\right)-\ln \left(\sqrt{x^{2}+(n-1)^{2} R^{2}}\right)\right)
\end{aligned}
$$

where, " $M$ " is the arbitrary point in the solid sphere, " $h$ " the point corresponding to " $M$ " horizontally. " $r$ " is the distance of the point " $M$ " from original point " $o$ ", $\rho$ ' is ideally homogeneous mass density of a solid sphere, $\rho$ the average one. $k$ stands for the calibration factor of average mass density.

For a general integral

$$
\begin{aligned}
& \int_{0}^{R} \mathrm{~d} x \ln \left(\sqrt{x^{2}+C}\right),\left(C=(n \pm 1)^{2} R^{2}\right)=\left.x \ln \left(\sqrt{x^{2}+C}\right)\right|_{0} ^{R} \int_{0}^{R} \mathrm{~d} x \frac{x^{2}}{x^{2}+C} \\
& =R \ln (R \sqrt{1+C})=R+\sqrt{C} \operatorname{arctg} \frac{R}{\sqrt{C}}
\end{aligned}
$$

For integral

$$
\begin{aligned}
& \int_{0}^{R} \mathrm{~d} x \ln \left(R+\sqrt{x^{2}+\left(1+(n \pm 1)^{2}\right) R^{2}}\right)=\left.x \ln \left(R+\sqrt{x^{2}+\left(1+(n \pm 1)^{2}\right) R^{2}}\right)\right|_{0} ^{R} \\
& -\int_{0}^{R} \mathrm{~d} x \frac{x^{2}}{R+\sqrt{x^{2}+\left(1+(n \pm 1)^{2}\right) R^{2}}} \frac{1}{\sqrt{x^{2}+\left(1+(n \pm 1)^{2}\right) R^{2}}}=\left.x \ln \left(R+\sqrt{x^{2}+\left(1+(n \pm 1)^{2}\right) R^{2}}\right)\right|_{0} ^{R} \\
& -\int_{0}^{R} \mathrm{~d} x\left(1+\frac{(n \pm 1)^{2} R}{R+\sqrt{x^{2}+\left(1+(n \pm 1)^{2}\right) R^{2}}}-\frac{\left(1+(n \pm 1)^{2}\right) R}{\sqrt{x^{2}+\left(1+(n \pm 1)^{2}\right) R^{2}}}\right) \\
& =R \ln \left(R+\sqrt{R^{2}+\left(1+(n \pm 1)^{2}\right) R^{2}}\right)-R+\left.\left(1+(n \pm 1)^{2}\right) R \ln \left(x+\sqrt{x^{2}+\left(1+(n \pm 1)^{2}\right) R^{2}}\right)\right|_{0} ^{R} \\
& -\int_{0}^{R} \mathrm{~d} x \frac{(n \pm 1)^{2} R}{R+\sqrt{x^{2}+\left(1+(n \pm 1)^{2}\right) R^{2}}} \int_{0}^{R} \mathrm{~d} x \ln \left(R+\sqrt{x^{2}+\left(1+(n \pm 1)^{2}\right) R^{2}}\right) \\
& =R \ln \left(R\left(1+\sqrt{\left(2+(n \pm 1)^{2}\right)}\right)\right)-R+\left(1+(n \pm 1)^{2}\right) R \ln \frac{1+\sqrt{2+(n \pm 1)^{2}}}{\sqrt{1+(n \pm 1)^{2}}} \\
& -\int_{0}^{R} \mathrm{~d} x \frac{(n \pm 1)^{2} R}{R+\sqrt{x^{2}+\left(1+(n \pm 1)^{2}\right) R^{2}}} .
\end{aligned}
$$


For general integral

$$
\int_{0}^{R} \mathrm{~d} x \frac{(n \pm 1)^{2} R}{A+\sqrt{x^{2}+B}},\left(A=R, B=\left(1+(n \pm 1)^{2}\right) R^{2}\right)
$$

Let $f=\left(A+\sqrt{x^{2}+B}\right)^{-1}$

$$
\begin{aligned}
& f(0)=\frac{1 / R}{1+\sqrt{1+(n \pm 1)^{2}}} . \\
& f^{\prime}=-\left(A+\sqrt{x^{2}+B}\right)^{-2} x\left(x^{2}+B\right)^{-1 / 2} \\
& f^{\prime \prime}=2 x^{2}\left(x^{2}+B\right)^{-1}\left(A+\sqrt{x^{2}+B}\right)^{-3}-\left(A+\sqrt{x^{2}+B}\right)^{-2}\left(\left(x^{2}+B\right)^{-1 / 2}-x^{2}\left(x^{2}+B\right)^{-3 / 2}\right) . \\
& f^{\prime \prime}(0)=\frac{1}{R^{3}} \frac{1}{\sqrt{1+(n \pm 1)^{2}}\left(1+\sqrt{1+(n \pm 1)^{2}}\right)^{2}} . \\
& f^{\prime \prime \prime}=-6 x^{3}\left(x^{2}+B\right)^{-3 / 2}\left(A+\sqrt{x^{2}+B}\right)^{-4}+2\left(A+\sqrt{x^{2}+B}\right)^{-3}\left(2 x\left(x^{2}+B\right)^{-1}-2 x^{3}\left(x^{2}+B\right)^{-2}\right) \\
& -\left(A+\sqrt{x^{2}+B}\right)^{-2}-\left(3 x\left(x^{2}+B\right)^{-3 / 2}+3 x^{3}\left(x^{2}+B\right)^{-5 / 2}\right) \\
& +2\left(\left(x^{2}+B\right)^{-1 / 2}-x^{2}\left(x^{2}+B\right)^{-3 / 2}\right)\left(A+\sqrt{x^{2}+B}\right)^{-3} x\left(x^{2}+B\right)^{-1 / 2} \text {. } \\
& f^{\prime \prime \prime \prime}=24 x^{4}\left(x^{2}+B\right)^{-4 / 2}\left(A+\sqrt{x^{2}+B}\right)^{-5} \\
& -6\left(A+\sqrt{x^{2}+B}\right)^{-4}\left(3 x^{2}\left(x^{2}+B\right)^{-3 / 2}-3 x^{4}\left(x^{2}+B\right)^{-5 / 2}\right)+2\left(A+\sqrt{x^{2}+B}\right)^{-3} \\
& \left(2\left(x^{2}+B\right)^{-1}-4 x^{2}\left(x^{2}+B\right)^{-2}-6 x^{2}\left(x^{2}+B\right)^{-2}+8 x^{4}\left(x^{2}+B\right)^{-3}\right) \\
& -\left(A+\sqrt{x^{2}+B}\right)^{-2}\left(-3\left(x^{2}+B\right)^{-3 / 2}+9 x^{2}\left(x^{2}+B\right)^{-5 / 2}-9 x^{2}\left(x^{2}+B\right)^{-5 / 2}-15 x^{4}\left(x^{2}+B\right)^{-7 / 2}\right) \\
& \left.+6 x^{2}\left(x^{2}+B\right)^{-1 / 2}\left(A+\sqrt{x^{2}+B}\right)^{-3}\left(x^{2}+B\right)^{-3 / 2}+3 x^{3}\left(x^{2}+B\right)^{-5 / 2}\right)+2\left(\left(x^{2}+B\right)^{-1 / 2}-x^{2}\left(x^{2}+B\right)^{-3 / 2}\right) \\
& \left(-3 x^{2}\left(x^{2}+B\right)^{-2 / 2}\left(A+\sqrt{x^{2}+B}\right)^{-4}+\left(A+\sqrt{x^{2}+B}\right)^{-3}\left(\left(x^{2}+B\right)^{-1 / 2}-x^{2}\left(x^{2}+B\right)^{-3 / 2}\right)\right) \\
& +2\left(A+\sqrt{x^{2}+B}\right)^{-3} x\left(x^{2}+B\right)^{-1 / 2} \times\left(-x\left(x^{2}+B\right)^{-3 / 2}-2 x\left(x^{2}+B\right)^{-3 / 2}+3 x\left(x^{2}+B\right)^{-5 / 2}\right) . \\
& f^{\prime \prime \prime \prime}(0)=\frac{1}{R^{5}}\left(\frac{6}{\left(1+(n \pm 1)^{2}\right)\left(1+\sqrt{1+(n \pm 1)^{2}}\right)^{3}}+\frac{3}{\left(1+(n \pm 1)^{2}\right)^{3 / 2}\left(1+\sqrt{1+(n \pm 1)^{2}}\right)^{2}}\right) .
\end{aligned}
$$




$$
\begin{aligned}
\int_{0}^{R} \mathrm{~d} x \frac{(n \pm 1)^{2} R}{A+\sqrt{x^{2}+B}} & =R^{\prime}(n \pm 1)^{2} \int_{0}^{R} \mathrm{~d} x\left(f(0)+\frac{f^{\prime \prime}(0)}{2 !} x^{2}+\frac{f^{\prime \prime \prime}(0)}{4 !} x^{4}+\cdots\right) \\
& =R^{\prime}(n \pm 1)^{2}\left(\frac{1 / R}{1+\sqrt{1+(n \pm 1)^{2}}} R+\frac{1}{3 \times 2 !} \frac{1}{R^{3}} \frac{1}{\sqrt{1+(n \pm 1)^{2}}\left(1+\sqrt{1+(n \pm 1)^{2}}\right)^{2}} R^{3}\right. \\
& \left.+\frac{1}{5 \times 4 !} \frac{1}{R^{5}}\left(\frac{6}{\left(1+(n \pm 1)^{2}\right)\left(1+\sqrt{1+(n \pm 1)^{2}}\right)^{3}}+\frac{3}{\left(1+(n \pm 1)^{2}\right)^{3 / 2}\left(1+\sqrt{1+(n \pm 1)^{2}}\right)^{2}}\right) R^{5}+\cdots\right) \\
& =R^{\prime}(n \pm 1)^{2}\left(\frac{1}{1+\sqrt{1+(n \pm 1)^{2}}+\frac{1}{6} \frac{1}{\sqrt{1+(n \pm 1)^{2}}\left(1+\sqrt{1+(n \pm 1)^{2}}\right)^{2}}}\right) \\
& \left.\left.+\frac{1}{40}\left(\frac{2}{\left(1+(n \pm 1)^{2}\right)\left(1+\sqrt{1+(n \pm 1)^{2}}\right)^{3}}+\frac{1}{\left(1+(n \pm 1)^{2}\right)^{3 / 2}\left(1+\sqrt{1+(n \pm 1)^{2}}\right)^{2}}\right)\right)^{(1}\right)
\end{aligned}
$$

Substituted (3) with (4)

$$
\begin{aligned}
& \int_{0}^{R} \mathrm{~d} x \ln \left(R+\sqrt{x^{2}+\left(1+(n \pm 1)^{2}\right) R^{2}}\right)=R \ln \left(R\left(1+\sqrt{\left(2+(n \pm 1)^{2}\right)}\right)\right) \\
& \left.-R+\left(1+(n \pm 1)^{2}\right) R \ln \frac{1+\sqrt{2+(n \pm 1)^{2}}}{\sqrt{1+(n \pm 1)^{2}}}-R^{\prime}(n \pm 1)^{2}\left(\frac{1}{1+\sqrt{1+(n \pm 1)^{2}}}+\frac{1}{6} \frac{1}{\sqrt{1+(n \pm 1)^{2}}\left(1+\sqrt{1+(n \pm 1)^{2}}\right)^{2}}\right)\right) \\
& \left.+\frac{1}{40}\left(\frac{2}{\left(1+(n \pm 1)^{2}\right)\left(1+\sqrt{1+(n \pm 1)^{2}}\right)^{3}}+\frac{1}{\left(1+(n \pm 1)^{2}\right)^{3 / 2}\left(1+\sqrt{1+(n \pm 1)^{2}}\right)^{2}}\right)\right)
\end{aligned}
$$

As the results of (2) and (5) expressed, thus, (1) becomes

$$
\begin{aligned}
a & =4 G k \rho R\left(\ln \left(R\left(1+\sqrt{2+(n-1)^{2}}\right)\right)-1\left(1+(n-1)^{2}\right) \ln \frac{1+\sqrt{2+(n-1)^{2}}}{\sqrt{1+(n-1)^{2}}}\right. \\
& -(n-1)^{2}\left(\frac{1}{1+\sqrt{1+(n-1)^{2}}}+\frac{1}{6} \frac{1}{\sqrt{1+(n-1)^{2}}\left(1+\sqrt{1+(n-1)^{2}}\right)^{2}}\right. \\
& \left.+\frac{1}{20} \frac{1}{\left(1+(n-1)^{2}\right)\left(1+\sqrt{1+(n-1)^{2}}\right)^{3}}+\frac{1}{40} \frac{1}{\left(1+(n-1)^{2}\right)^{3 / 2}\left(1+\sqrt{1+(n-1)^{2}}\right)^{2}}\right) \\
& -\ln \left(R\left(1+\sqrt{2+(n-1)^{2}}\right)\right)+1-\left(1+(n-1)^{2}\right) \ln \frac{1+\sqrt{2+(n-1)^{2}}}{\sqrt{1+(n-1)^{2}}}
\end{aligned}
$$




$$
\begin{aligned}
& +(n-1)^{2}\left(\frac{1}{1+\sqrt{1+(n-1)^{2}}}+\frac{1}{6} \frac{1}{\sqrt{1+(n-1)^{2}}\left(1+\sqrt{1+(n-1)^{2}}\right)^{2}}\right. \\
& \left.+\frac{1}{20} \frac{1}{\left(1+(n-1)^{2}\right)\left(1+\sqrt{1+(n-1)^{2}}\right)^{3}}+\frac{1}{40} \frac{1}{\left(1+(n+1)^{2}\right)^{3 / 2}\left(1+\sqrt{1+(n+1)^{2}}\right)^{2}}\right) \\
& \left.+\ln \left(R \sqrt{1+(n+1)^{2}}\right)-1+(n+1) \operatorname{arctg} \frac{1}{(n+1)}-\ln \left(R \sqrt{1+(n-1)^{2}}\right)+1-|n-1| \operatorname{arctg} \frac{1}{|n-1|}+\cdots\right) \text {. }
\end{aligned}
$$

Let $a(n)=4 G k \rho R F(n)$, then,

$$
\begin{aligned}
F(n)= & \left(\ln \frac{1+\sqrt{2+(n-1)^{2}}}{1+\sqrt{2+(n+1)^{2}}}+\left(1+(n-1)^{2}\right) \ln \frac{1+\sqrt{2+(n-1)^{2}}}{\sqrt{1+(n-1)^{2}}}-\left(1+(n+1)^{2}\right) \ln \frac{1+\sqrt{2+(n+1)^{2}}}{\sqrt{1+(n+1)^{2}}}\right. \\
& -(n-1)^{2}\left(\frac{1}{1+\sqrt{1+(n-1)^{2}}}+\frac{1}{6} \frac{1}{\sqrt{1+(n-1)^{2}}\left(1+\sqrt{1+(n-1)^{2}}\right)^{2}}\right. \\
& \left.+\frac{1}{20} \frac{1}{\left(1+(n-1)^{2}\right)\left(1+\sqrt{1+(n-1)^{2}}\right)^{3}}+\frac{1}{40} \frac{1}{\left(1+(n-1)^{2}\right)^{3 / 2}\left(1+\sqrt{1+(n-1)^{2}}\right)^{2}}\right) \\
& +(n+1)^{2}\left(\frac{1}{1+\sqrt{1+(n+1)^{2}}}+\frac{1}{6} \frac{1}{\sqrt{1+(n+1)^{2}}\left(1+\sqrt{1+(n+1)^{2}}\right)^{2}}\right. \\
& \left.+\frac{1}{20} \frac{1}{\left(1+(n+1)^{2}\right)\left(1+\sqrt{1+(n+1)^{2}}\right)^{3}}+\frac{1}{40} \frac{1}{\left(1+(n+1)^{2}\right)^{3 / 2}\left(1+\sqrt{1+(n+1)^{2}}\right)^{2}}\right) \\
& +\ln \frac{\sqrt{1+(n+1)^{2}}}{\sqrt{1+(n-1)^{2}}+|n+1| \operatorname{arc} \operatorname{tg} \frac{1}{|n+1|}-|n+1| \operatorname{arctg} \frac{1}{|n+1|} .}
\end{aligned}
$$

$F(n)$ is a function of $n$. The, $F-n$, diagram was showed in Figure 2.

For the earth, the average mass density $\rho$ is $5.5153 \times$ $10^{3} \mathrm{~kg} \cdot \mathrm{m}^{-3}$, the radius $R$ is $6.356078 \times 10^{6} \mathrm{~m}$. The gravitational acceleration on earth surface was calculated $(n=$ 1) to be $12.68 \mathrm{~m} \cdot \mathrm{s}^{-2}$. However, the real value of gravitational acceleration on earth is $9.81 \mathrm{~m} \cdot \mathrm{s}^{-2}$. It is 0.7736 times than the calculated one. It is because of the earth that does not have an ideally homogeneous density used in above formula derivation process. The ratio can be used as calibration factor, $k$, of average density of a solid sphere $\left(\rho^{\prime}=k \rho\right.$. For earth, $k=0.7736$; and it was sup- posed to be fit for any other celestial bodies).

\subsection{Formula Derivation B-Self-Gravitational Pressure in Center of Solid Sphere}

The gravitational pressure in center from solid sphere can be calculated according to Equation (6).

$$
\mathrm{d} F=a \mathrm{~d} m=a \rho_{1} \mathrm{~d} V=\rho_{1} S R a \mathrm{~d} n / 3,(\text { where, } V=S n R / 3 \text { ) }
$$$$
d p=\rho_{1} \operatorname{SRadn} / 3
$$

$$
p=\int \mathrm{d} p=\frac{4}{3} \rho_{1} G k \rho_{2} R^{2} \int_{0}^{1} F(n) \mathrm{d} n .
$$


The integral, $\int_{0}^{1} F(n) \mathrm{d} n$, can be calculated diagrammatically. The diagram showed in Figure 3.

The area beneath the curve calculated by computer gave the result of 0.62129 . Thus, $p=0.62129 \times$ $4 G k \rho^{2} R^{2} / 3$. Supposing the considered celestial body has the same value of calibration factor $k$ of earth, then we obtained

$$
p=0.6408 G \rho^{2} R^{2} .
$$

\subsection{Formula Derivation C-Gravitational Potential Energy from Solid Sphere}

The gravitational potential energy, $E_{\mathrm{g}}$, from a solid sphere can be calculated.

$$
E_{g}=\int \mathrm{d} E_{\mathrm{g}}=\int m a \mathrm{~d} l=4 m G k \rho R^{2} \int_{n}^{\infty} F(n) \mathrm{d} n .
$$

According to relation (6), the integral, $\int_{1}^{\infty} F(n) \mathrm{d} n$, can be calculated diagrammatically. The diagram showed in Figure 4.

The area beneath the curve calculated by computer

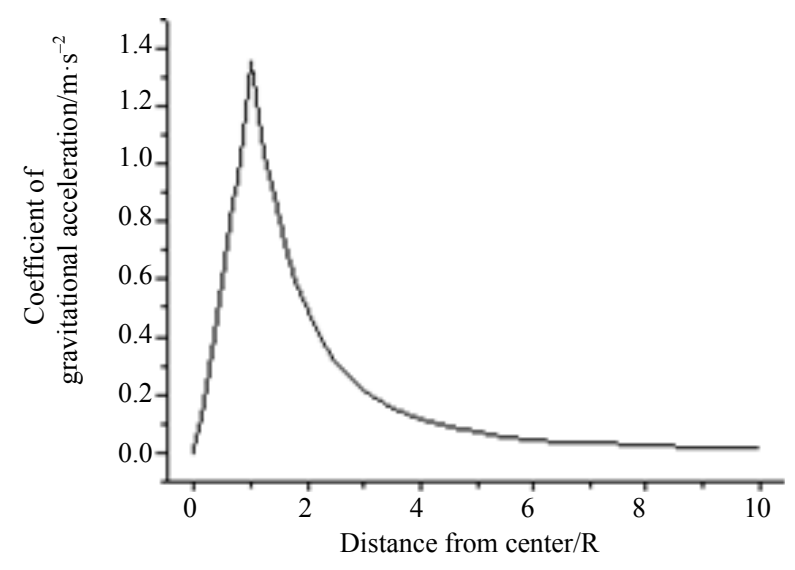

Figure 2. Diagram of function $F(n)$.

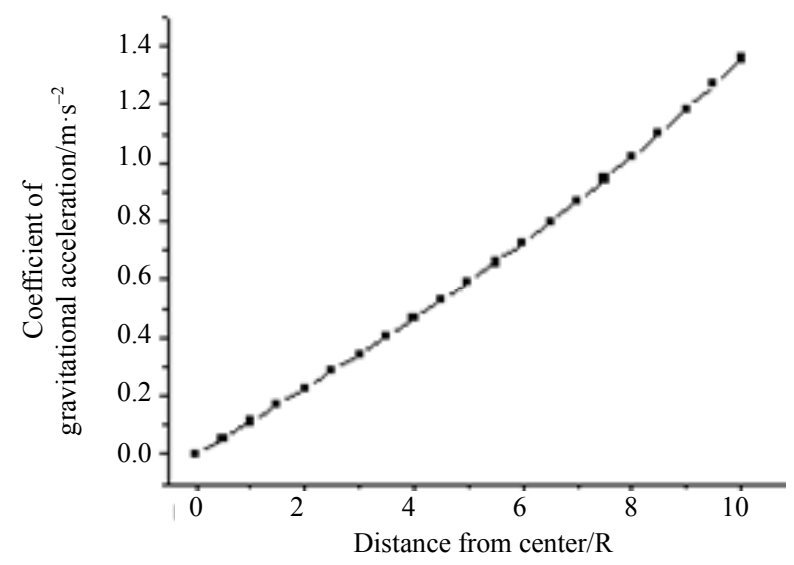

Figure 3. Partial diagram of function $F(n)$. The variable $n$ changes from zero to one.

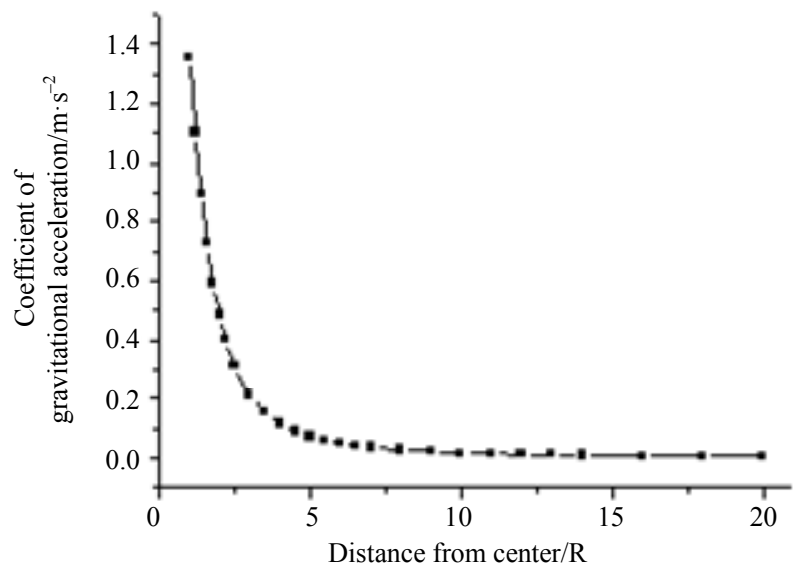

Figure 4. Partial diagram of function $F(n)$. The variable $n$ changes from 1 to $\infty$.

gave the result of 1.7193. Therefore, we have

$$
E_{\mathrm{g}}=1.7193 \times 4 G \mathrm{k \rho m} R^{2}=5.320 G \rho m R^{2} .
$$

\section{Target Calculation}

\subsection{Maximal Radius of Celestial Bodies}

Atoms have similar energy demand when one electron is compressed in nucleus forming a new atom (which has lower atomic number). Taking atom zinc as an example, when one electron of $\mathrm{Zn}$ is compressed in nucleus, the isotopic atom $\mathrm{Cu}$ is then formed. The energy demand, $\Delta E_{\mathrm{a}}$, can be calculated. According to quantum theory, the energy of an outer electron of a atom has the form $\mathrm{E} \approx$ $-13.6 \mathrm{Z}^{2} / n^{2}$ (in ground state). Thus,

$$
\begin{aligned}
& E_{Z n}=-13.6 \frac{Z^{2}}{n^{2}}=-13.6 \frac{30^{2}}{4^{2}}=-765 \mathrm{eV} . \\
& E_{c u}=-13.6 \frac{Z^{2}}{n^{2}}=-13.6 \frac{29^{2}}{4^{2}}=-715 \mathrm{eV} . \\
& \Delta E_{1}=E_{c u}-E_{Z n}=50 \mathrm{eV} .
\end{aligned}
$$

For an extreme hot electron of atom of hydrogen,

$$
\Delta E_{\mathrm{a}}=\Delta E_{1}+13.6=63.6 \mathrm{eV}=1.02 \times 10^{-17} \mathrm{~J}
$$

As the gravitational force increasing, many radioactive isotopes formed with the atomic number decreasing. Considering the atom of hydrogen, that has the maximum compression radius $a_{0}$, we guessed that the hydrogen atoms would be the last element to be compressed to nucleus. Then, the maximum pressure, $p_{\max , \mathrm{a}}$ for compressing a hydrogen atom to neutron could be derived as:

$$
\begin{aligned}
\Delta E_{\mathrm{a}}= & F \Delta r=p S \Delta r=p S a_{0} . \\
p_{\text {max }, a} & =\Delta E a / V=\frac{3 \Delta E_{a}}{4 \pi a_{0}{ }^{3}}=\frac{3 \times 1.02 \times 10^{-17}}{3.14159 \times 4 \times\left(5.29 \times 10^{-11}\right)^{3}} \\
& =1.64 \times 10^{13} \mathrm{Nm}^{-2}
\end{aligned}
$$


where, $\Delta r$ was replaced with the radius of hydrogen atom $a_{0 .}$

The pressure comes from the gravitation of celestial body. Combining Equations (7.1) and (9), we have

$$
0.6408 G \rho^{2} R^{2}=1.64 \times 10^{13},
$$

then,

$$
R_{\max }=5.07 \times 10^{6} \frac{1}{\rho \sqrt{G}} .
$$

If a solid celestial body has the same average mass density of earth (5.5153), then

$$
\begin{aligned}
R_{\max , \text { solid }} & =5.07 \times 10^{6} \frac{1}{5.5153 \times 10^{3} \times \sqrt{6.670 \times 10^{-11}}} \\
& =1.12 \times 10^{8} \mathrm{~m} .
\end{aligned}
$$

To a hot gas celestial body, which has the same average mass density of sun (1.409), then, we have

$$
\begin{aligned}
R_{\text {max } \text { gas }} & =5.07 \times 10^{6} \frac{1}{1.409 \times 10^{3} \times \sqrt{6.670 \times 10^{-11}}} \\
& =4.41 \times 10^{8} \mathrm{~m} . \\
m_{\max }= & \rho V=5.06 \times 10^{29} \mathrm{~kg} .
\end{aligned}
$$

\subsection{Minimum Neutron Star}

A model of neutron star was showed in Figure 5. A neutron body with the radius of $R$ is in the center of the star. A general matter layer with the thickness of $(n-1) R$ is around the neutron body.

The gravitational pressure on the surface of neutron body can be calculated. It contains two parts.

Equation (6) can express linearly in a short segment.

$$
F(n)=1.355571 n,(n=0 \rightarrow 1) .
$$

According to Equation (7),

$$
\begin{gathered}
p_{i}=\int \mathrm{d} p=\frac{4}{3} \rho_{1} G k p_{2} R^{2} \int F(n) \mathrm{d} n . \\
p_{1}=\frac{4}{3} \rho_{1} G k p_{2} \int_{1}^{n} F(n) \mathrm{d} n=\frac{4}{3} \rho^{2} G k R^{2} \\
=\int_{1}^{n} 1.355571 n \mathrm{~d} n=0.9037 \rho^{2} k G\left(n^{2}-1\right) R^{2} . \\
p_{2}=\frac{4}{3} \rho_{1} G k \rho_{2} R^{2} \int_{1}^{n} F(n) \mathrm{d} n \\
=\frac{4}{3} \rho \rho_{n} k G R^{2} \int_{1}^{n} F(n) \mathrm{d} n . \\
p=p_{1}+p_{2} .
\end{gathered}
$$

where, $\rho_{\mathrm{n}}$ is the nuclear mass density, $\rho$ the mass density layer matter. Substituting the parameters, we have

$$
\begin{aligned}
p & =1.1418 \times 10^{-3}\left(n^{2}-1\right) R^{2} \\
& +7.589 \times 10^{10} R^{2} \int_{1}^{n} F(n) \mathrm{d} n
\end{aligned}
$$

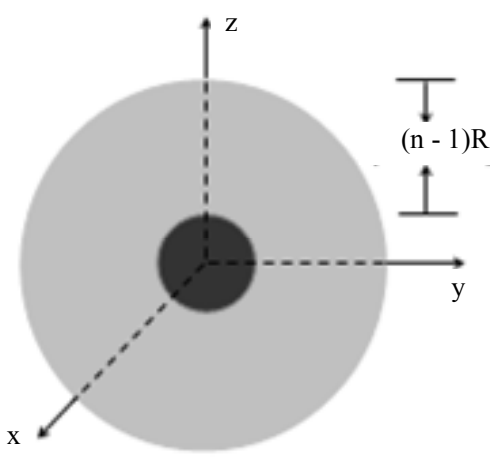

Figure 5. A model of neutron star.

Combining Equations (9) and (10), we have

$1.1418 \times 10^{-3}\left(n^{2}-1\right) R^{2}+7.589 \times 10^{10} R^{2} \int_{1}^{n} F(n) \mathrm{d} n$

$=1.64 \times 10^{13}$.

According to Equations (6) and (11), we obtained following results:

$$
\begin{aligned}
& R=11.2 \mathrm{~m}, n R=1121 \mathrm{~m} \\
& R=16 \mathrm{~m}, n R=32 \mathrm{~m} \\
& R=261 \mathrm{~m}, n R=261.6 \mathrm{~m} \\
& R=15257 \mathrm{~m}, n R=15257 \mathrm{~m} .
\end{aligned}
$$

Combining Equations (7.1) and (13), we have

$$
\begin{aligned}
R & =\sqrt{\frac{1.99 \times 10^{32}}{0.6408 G \rho_{n}^{2}}} \\
& =\sqrt{\frac{1.99 \times 10^{32}}{0.6408 \times 6.670 \times 10^{-11} \times\left(2 \times 10^{17}\right)^{2}}} \\
& =15257 \mathrm{~m} . \\
m_{\max } & =p_{n} V=2.98 \times 10^{30} \mathrm{~kg} .
\end{aligned}
$$

\subsection{Minimal Mass of Black Hole}

When the radius of a neutron star achieved its maximal value, as the mass continuing accumulating, a graviton body began growing, the radius of neutron star then decreased correspondingly. When the mass of graviton body grew large enough, the neutron star began to have the ability to draw back a photon; we call it becoming a body of black hole. The maximum radius of black hole can be calculated by imitating the process of what done in paragraph 3.2. Just replacing $\rho$ and $\rho_{n}$ with the value of $\rho_{n}$ and $\rho_{g}$ (showed in Equation (20)), Equation (11) became Equation (12). Where $\rho_{g}$ is stands for the mass density of graviton.

$$
\begin{aligned}
p= & 1.865234 \times 10^{24}\left(n^{2}-1\right) R^{2} \\
& +8.682 \times 10^{24} R^{2} \int_{1}^{n} F(n) \mathrm{d} n .
\end{aligned}
$$

According to Equations (12) and (13), following rela- 
tions were obtained:

$$
\begin{aligned}
& R=0.971 \mathrm{~m}, n R=9714 \mathrm{~m}, \mathrm{M}=7.679 \times 10^{29} \mathrm{~kg} \\
& R=9.714 \mathrm{~m}, n R=9714 \mathrm{~m}, \mathrm{M}=7.679 \times 10^{29} \mathrm{~kg} \\
& R=97.10 \mathrm{~m}, n R=9710 \mathrm{~m}, \mathrm{M}=7.671 \times 10^{29} \mathrm{~kg} \\
& R=1154 \mathrm{~m}, n R=9279 \mathrm{~m}, \mathrm{M}=6.722 \times 10^{29} \mathrm{~kg} \\
& R=4078 \mathrm{~m}, n R=7340 \mathrm{~m}, \mathrm{M}=4.537 \times 10^{29} \mathrm{~kg} \\
& R=10793 \mathrm{~m}, n R=11873 \mathrm{~m}, \mathrm{M}=3.672 \times 10^{30} \mathrm{~kg} \\
& R=14090 \mathrm{~m}, n R=14908 \mathrm{~m}, \mathrm{M}=7.825 \times 10^{30} \mathrm{~kg} \\
& R=42004 \mathrm{~m}, n R=42273 \mathrm{~m}, \mathrm{M}=1.971 \times 10^{32} \mathrm{~kg} \\
& R=74934 \mathrm{~m}, n R=75084 \mathrm{~m}, \mathrm{M}=1.114 \times 10^{33} \mathrm{~kg} .
\end{aligned}
$$

\subsection{Calculation of Graviton}

Nuclear has the combining energy of about $8 \mathrm{MeV}$ (Wichmann 1971). Deducting the influence of proton, the maximum combining energy of nuclear would be 9.15 $\mathrm{MeV}$, i.e., $1.47 \times 10^{-12} \mathrm{~J}$. The parameter of the radius of a graviton, $r_{0}$, is the first needs to obtain. Depending on above suppositions described in Section 2.1, and the normal gravitational potential energy formula, $E=G m_{1} m_{2} / r$, substituting $m_{1}, m_{2}$ with the mass of neutron, $E$ with the combining energy in nucleus, then we have,

$$
\begin{aligned}
r_{0} & =G m_{1} m_{2} / E \\
& =6.670 \times 10^{-11} \times\left(1.67495 \times 10^{-27}\right)^{2} /\left(1.47 \times 10^{-12}\right) \\
& =1.273 \times 10^{-52} \mathrm{~m} .
\end{aligned}
$$

Supposing the energy demand for destroy a neutron, $\Delta E_{\mathrm{n}}$, is equal to its maximum combining energy, then we have

$$
\Delta E_{\mathrm{n}}=1.47 \times 10^{-12} \mathrm{~J} .
$$

Following the derivate process in Section 3.1, but replaced $\Delta E_{\mathrm{a}}$ with $\Delta E_{\mathrm{n}}, a_{0}$ with $r_{\mathrm{n}}$, Equation (9) becomes

$$
\begin{aligned}
p_{\max , n} & =\Delta E n / V=\frac{3 \Delta E_{n}}{4 \pi r_{n}^{3}} \\
& =\frac{3 \times 1.47 \times 10^{-12}}{4 \times 3.14159 \times\left(1.2598 \times 10^{-15}\right)^{3}} \\
& =1.76 \times 10^{32} \mathrm{Nm}^{-2} .
\end{aligned}
$$

Following the derivate process in Section 3.2, but replaced $\rho$ and $\rho_{n}$ with $\rho_{n}$, and $\rho_{\mathrm{g}}, p_{\max , a}$ with $p_{\max , n}$, Equation (11) became Equation (14).

$$
\begin{aligned}
& 1.865 \times 10^{24}\left(n^{2}-1\right) R^{2}+1.376 \times 10^{7} \rho_{g} R^{2} \int_{1}^{n} F(n) \mathrm{d} n \\
= & 1.99 \times 10^{-32} \\
R= & \left(1.76 \times 10^{32} /\left(1.865 \times 10^{24}\left(n^{2}-1\right)\right.\right. \\
& \left.\left.+1.376 \times 10^{7} \rho_{g} \int_{1}^{n} F(n) \mathrm{d} n\right)\right)^{1 / 2} .
\end{aligned}
$$

A Smallest Known Black Hole (named XTE J1650500 ) has the mass, $M_{\mathrm{b}}$, of $7.558 \times 10^{30} \mathrm{~kg}$ (Robert \& Rob 2008). Perhaps it is not the minimal black hole theoretically; however, it was considered to be true here temporarily. According to the data and Equation (8), we have

$$
\begin{gathered}
E_{g}=1.7193 \times 4 G k p_{n} m n^{2} R^{2} \\
+4 m G k\left(\rho_{g}-\rho_{n}\right) R^{2} \int_{n}^{\infty} F(n) \mathrm{d} n \\
=m c^{2} / 2 . \\
3.4386 \times 10^{17} n^{2} R^{2}+\left(\rho_{g}-\rho_{n}\right) R^{2} \int_{n}^{\infty}(n) \mathrm{d} n=2.180 \times 10^{26} \\
R=\left(2.180 \times 10^{26} /\left(3.4386 \times 10^{17}\right.\right. \\
\left.\left.+\left(\rho_{g}-2 \times 10^{17}\right) \int_{n}^{\infty} F(n) \mathrm{d} n\right)\right) . \\
M_{b}=2 \times 10^{17} \times\left(4 \pi n^{3} R^{3} / 3\right)+\left(\rho_{g}-\rho_{n}\right) \times\left(4 \pi R^{3} / 3\right) \\
=7.558 \times 10^{30} \cdot \\
2 \times 10^{17} \times\left(4 \pi n^{3} R^{3} / 3\right)+\left(\rho_{g}-\rho_{n}\right) \times\left(4 \pi R^{3} / 3\right) \\
=7.558 \times 10^{30} . \\
8.37766 \times 10^{17} n^{3} R^{3}+4.1888\left(\rho_{g}-\rho_{n}\right) R^{3} \\
=7.558 \times 10^{30} . \\
R=\left(7.558 \times 10^{30} /\left(8.3776 \times 10^{17} n^{3}\right.\right. \\
\left.\left.+4.1888\left(\rho_{g}-2 \times 10^{17}\right)\right)\right)^{1 / 3} .
\end{gathered}
$$

Combining Equations (15), (16) and (17), we have Equations (18) and (19).

$$
\begin{aligned}
R & =\left(2.180 \times 10^{26} /\left(3.4386 \times 10^{17} n^{2}\right.\right. \\
& \left.\left.+\left(\rho_{g}-2 \times 10^{17}\right) \int_{n}^{\infty}(n) \mathrm{d} n\right)\right)^{1 / 2} \\
& =\left(7.558 \times 10^{30} /\left(8.3776 \times 10^{17} n^{3}\right.\right. \\
& \left.\left.+4.1888 .\left(\rho_{g}-2 \times 10^{17}\right)\right)\right)^{1 / 3} \\
& \left(2.180 \times 10^{26} /\left(3.4386 \times 10^{17} n^{2}\right.\right. \\
+ & \left.\left.\left(\rho_{g}-2 \times 10^{17}\right) \int_{n}^{\infty}(n) \mathrm{d} n\right)\right)^{1 / 2} \\
= & \left(1.76 \times 10^{32} /\left(1.865 \times 10^{24}\left(n^{2}-1\right)\right.\right. \\
& \left.\left.+1.376 \times 10^{7} \rho_{g} \int_{n}^{\infty}(n) \mathrm{d} n\right)\right)^{1 / 2} .
\end{aligned}
$$

Combining Equations (18) and (19) diagrammatically, we obtained

$$
\begin{aligned}
& n=1.0190 . \\
& R=14090 \mathrm{~m} .
\end{aligned}
$$




$$
\begin{gathered}
n R=14358 \mathrm{~m} . \\
\rho_{\mathrm{g}}=6.3096 \times 10^{17} \mathrm{~kg} \cdot \mathrm{m}^{-3} . \\
m_{g}=\rho_{g}\left(4 \pi r_{0}^{3} / 3\right)=6.3096 \times 10^{17} \\
\times\left(4 \pi\left(1.273 \times 10^{-52}\right)^{3} / 3\right)=5.452 \times 10^{-138} \mathrm{~kg} .
\end{gathered}
$$

where $m_{g}$ is stands for the mass of a graviton.

\section{Evolvement of the Universe}

The evolutive process of the universe was divided by us into six parts; and the process goes a circle $(\mathrm{a} \rightarrow \mathrm{f} \rightarrow \mathrm{a})$.

a) Growing of normal celestial body. Preponderant normal celestial bodies will attract substances from its surroundings. It gradually grows as it accumulates the mass. It would have the maximum mass of $5.06 \times 10^{29} \mathrm{~kg}$, radius, $4.41 \times 10^{8} \mathrm{~km}$.

b) Formation of neutron star. As the continuously accumulating of matter, the normal molecular celestial body begins to compress accompanied with neutron body engendered in the center. The compressing process looks like a process of phase variation. In the process, it will release large quantity of energy (lose of masses), become an extremely hot body and shine. A maximum neutron star has a maximum mass of $2.98 \times 10^{30} \mathrm{~kg}$, maximum radius of $15.3 \mathrm{~km}$.

c) Growing of graviton star. With continuously compressing, a graviton body would begin to grow in the center. The graviton star has a critical mass of $7.558 \times$ $10^{30} \mathrm{~kg}$, radius of $14.9 \mathrm{~km}$.

d) Formation of black hole. When graviton star grows to its critical value, it becomes a minimum black hole in the mean time. It can capture photons just near its surface. Afterward, it gradually grows in mass with the accumulating of matter. As the mass increasing, far more distant photons can be hold in. The compressing energy that released absorbed by itself. As an isolated system, the entropy of the black hole decreased spontaneously in its growing process.

e) Explosion of black hole. When the density of black hole increased large enough, the distance between gravitons going over a critical small value, the gravitational mechanism destroyed. The gravitation becomes repulsive force. Then, explosion occurred; the entropy increased sharply. Neutrons, protons, electrons, etc. particles and afterward atoms were produced subsequently in the explosion process. After explosion, this part of universe exists in expansion state in a unabiding period.

f) Constringency of partial universe. In the late period of explosion, the running matters slowed down, spread in wide space, mixed, and became part of cosmic dusts. Then, partial universe would exist in a contractive state in a long run. In intermediate constringency, the celestial bodies formed.

\section{Discussion}

When a celestial body has abundant material source in its surroundings, it will grow quickly and has a larger mass than we calculated. If the celestial body exists in process $b$, it would be a fixed star. The larger mass it has, the more quickly it will be compressed, and more efficiency the energy would release. i.e., the larger mass it owns, the higher brightness it will has. In this way, a shining star could burn with no existence of special fuels of nuclear fusion, but merely existence of normal atoms.

According to the calculated results in this paper, it is evident that every galaxy will have a big black hole in its center.

The universe consists of vast cosmic dusts and galaxies. They go along circle evolutive processes described above. Occasionally a limit big black hole explodes, causing the expansion in a partial space. However, in a long time scale, most space contracted slowly. The universe exists in a mobile equilibrium state of explosion and constringency (Thomas \& Hermann 1948). Those observed stars, which leave away at acceleratory velocity (Hubble 1929), might result in other reasons. It is by no means that the whole universe is expanding now. Reversely, our surrounding universe showed us its contractive views. We cannot imagine that the shape of our Milky Galaxy is formed in an expansion process.

\section{Tags}

A roughly information about graviton was derived in this paper. It is an exiting result to the researchers in fundamental physics. Future observation of existence of new smaller black hole is apparently meaningful.

\section{REFERENCES}

[1] V. L. Ginsburg, "On Physics and Astrophysics," Nauka Press, 1985.

[2] G. Erdei, "Principles of Structure of Matter," Mir Press, 1971.

[3] E. H. Wichmann, "Quantum Physics," Berkeley Physics Course, McGraw Hill, 1971.

[4] N. Robert and G. Rob, "NASA Scientists Identify Smallest Known Black Hole," NASA's Goddard Space Flight Center, 2008.

http://www.nasa.gov/centers/goddard/news/topstory/2008 /smallest_blackhole.html

[5] G. Thomas and B. Hermann, "The Steady-State Theory of the Expanding Universe," Royal Astronomical Society, Vol. 108, 1948, p. 252.

[6] E. Hubble, "A Relation between Distance and Radial Velocity among Extra-Galactic Nebulae," Proceedings of the National Academy of Sciences, Vol. 15, 1929, p. 16. 